\title{
Micro-Computed Tomographical Imaging of Soft Biological Materials Using Contrast Techniques
}

\author{
Kaeuis A. Faraj, Ph.D., ${ }^{1,2}$ Vincent M.J.I. Cuijpers, B.Sc., ${ }^{3}$ \\ Ronnie G. Wismans, M.Sc., ${ }^{1}$ X. Frank Walboomers, Ph.D., ${ }^{3}$ John A. Jansen, Ph.D., ${ }^{3}$ \\ Toin H. van Kuppevelt, Ph.D., ${ }^{1}$ and Willeke F. Daamen, Ph.D. ${ }^{1}$
}

The aim of this work was to introduce high-resolution computed tomography (micro-CT) for scaffolds made from soft natural biomaterials, and to compare these data with the conventional techniques scanning electron microscopy and light microscopy. Collagen-based scaffolds were used as examples. Unlike mineralized tissues, collagen scaffolds do not provide enough X-ray attenuation for micro-CT imaging. Therefore, various metalbased contrast agents were applied and evaluated using two structurally distinct scaffolds, one with round pores and one with unidirectional lamellae. The optimal contrast techniques for obtaining high-resolution threedimensional images were either a combination of osmium tetroxide and uranyl acetate, or a combination of uranyl acetate and lead citrate. The data obtained by micro-CT analysis were in line with data obtained by light and electron microscopy. However, small structures (less than a few $\mu \mathrm{m}$ ) could not be visualized due to limitation of the spot size of the micro-CT apparatus. In conclusion, reliable three-dimensional images of scaffolds prepared from soft natural biomaterials can be obtained using appropriate contrast protocols. This extends the use of micro-CT analysis to soft materials, such as protein-based biomaterials.

\section{Introduction}

$\mathbf{T}$ HE DEVELOPMENT OF High-RESOlUtion X-ray computed tomography (micro-CT) started in the early 1980s and has been used extensively to study the structure and architecture of bone tissue. ${ }^{1-5}$ Various parameters can be calculated with this technique, depending on the computational capability of the hardware and software. In addition, microCT is basically a nondestructive technique. ${ }^{6}$

Researchers have employed micro-CT in the field of tissue engineering. ${ }^{6-8}$ The versatility of micro-CT has been demonstrated in the evaluation of scaffolds, because this single technique is capable of characterizing multiple aspects of the scaffolds. ${ }^{6}$ Micro-CT enables to get three-dimensional (3D) images of the internal area of a sample, and a detailed 3D view of pores at any depth. ${ }^{9}$ Further, different parameters may be calculated such as porosity, surface area to volume ratio, pore size, pore wall thickness, anisotropy, crosssectional area, and permeability. ${ }^{6}$ Micro-CT has been used for several polymer-based scaffolds that hold sufficient intrinsic contrast. For example, the internal geometry, pore network, and pore interconnectivity of poly- $\varepsilon$-caprolactone scaffolds have been determined ${ }^{10}$; in addition, the porosity, surface area to volume ratio, and interconnectivity of scaffolds made from a copolymer of poly ethylene glycol, poly- $\varepsilon-$ caprolactone, and polylactic acid have been evaluated. ${ }^{6}$ Quantification of microarchitectural parameters, including volume fraction, density, thickness, spacing, and degree of anisotropy, of porous poly(L-lactide-co-DL-lactide) scaffolds with axially oriented macroporosity and random microporosity has also been reported. ${ }^{7}$

Scaffolds based on natural proteins such as collagen do not have the intrinsic X-ray attenuation capacity to be imaged by 3D micro-CT. Consequently, additional contrast has to be imposed upon such scaffolds. The aim of this work was to introduce micro-CT for proteinaceous scaffolds as exemplified by collagen, and to compare results with those obtained by scanning electron microscopy and light microscopy. Two structurally different scaffold types were tested, one with round pores and one with unidirectional lamellae. Different heavy metal contrast agents were assessed to find useful radio-opaque contrast agents that allow the use of micro-CT imaging for collagen scaffolds in particular and protein-based scaffolds in general.

\footnotetext{
${ }^{1}$ Department of Biochemistry 280, Nijmegen Centre for Molecular Life Sciences, Radboud University Nijmegen Medical Centre, Nijmegen, The Netherlands.

${ }^{2}$ aap Bioimplant-EMCM BV, Nijmegen, The Netherlands.

${ }^{3}$ Department of Biomaterials 309, Radboud University Nijmegen Medical Centre, Nijmegen, The Netherlands.
} 


\section{Materials and Methods}

\section{Collagen scaffolds}

Collagen scaffolds were prepared as previously described. ${ }^{11}$ Briefly, a collagen suspension was prepared by incubation of $0.9 \%$ purified insoluble type I collagen in $0.25 \mathrm{M}$ acetic acid at $4{ }^{\circ} \mathrm{C}$ for $16 \mathrm{~h}$. The suspension was homogenized on ice, followed by deaeration by centrifugation at $525 g$, resulting in the collagen suspension to be used for the scaffold.

Scaffolds with round pores were prepared by freezing $4 \mathrm{~mL}$ of collagen suspension per well of a six-well culture plate at $-20^{\circ} \mathrm{C}$. Scaffolds with unidirectional lamellae connected by thin struts (about $1-2 \mu \mathrm{m}$ ) were prepared using a temperature gradient between liquid nitrogen $\left(-196^{\circ} \mathrm{C}\right)$ and ambient temperature. This induces a temperature gradient starting from the side of $\mathrm{N}_{2}$ (l) toward the ambient temperature, thus producing lamellar structures. The principle is essentially the same as described by Schoof et al. ${ }^{12}$ The frozen collagen suspensions were then lyophilized to obtain dry scaffolds. Samples of approximately $5 \times 5 \times 5 \mathrm{~mm}$ were used for analysis.

\section{Scaffold preparation for imaging}

To obtain collagen scaffolds with appropriate contrast for micro-CT imaging, different contrast agents were used: osmium tetroxide, uranyl acetate, and lead citrate. The stains were selected based on contrast agents used in electron microscopy: first, because they bind to biological materials, and, second, because by being heavy metals they have the ability to apply contrast. In particular, uranyl acetate and lead citrate are used to stain collagen for electron microscopy. ${ }^{13}$ All scaffolds were treated with different contrast agents in distilled water at $22^{\circ} \mathrm{C}$, followed by three $20 \mathrm{~min}$ washings in distilled water. The applied procedures were as follows:

(1) $1 \%(\mathrm{w} / \mathrm{v})$ osmium tetroxide for $24 \mathrm{~h}$;

(2) $1 \%(\mathrm{w} / \mathrm{v})$ osmium tetroxide for 6 days;

(3) $1 \%(\mathrm{w} / \mathrm{v})$ lead citrate for $24 \mathrm{~h}$;

(4) $2 \%(\mathrm{w} / \mathrm{v})$ uranyl acetate for $24 \mathrm{~h}$;

(5) $2 \%(\mathrm{w} / \mathrm{v})$ uranyl acetate for $24 \mathrm{~h}$, followed by three washings in distilled water for $20 \mathrm{~min}$ and $1 \%$ $(\mathrm{w} / \mathrm{v})$ lead citrate for $24 \mathrm{~h}$;

(6) $1 \%(\mathrm{w} / \mathrm{v})$ osmium tetroxide for 6 days, followed by three washings in distilled water for $20 \mathrm{~min}$ and $1 \%(\mathrm{w} / \mathrm{v})$ lead citrate for $24 \mathrm{~h}$;

(7) $1 \%(\mathrm{w} / \mathrm{v})$ osmium tetroxide for 6 days, followed by three washings in distilled water for $20 \mathrm{~min}$ and $2 \%(\mathrm{w} / \mathrm{v})$ uranyl acetate for $24 \mathrm{~h}$;

(8) $1 \%(\mathrm{w} / \mathrm{v})$ osmium tetroxide for 6 days, rinsed five times for $15 \mathrm{~min}$ with distilled water, followed by $1 \%(\mathrm{w} / \mathrm{v})$ thiocarbohydrazide for $1 \mathrm{~h}$, rinsed five times for $15 \mathrm{~min}$ with distilled water, and $1 \%$ osmium tetroxide for $1 \mathrm{~h}$ (treatment according to Kelly et al. ${ }^{14}$ for enhanced contrast intensity due to increased binding of osmium tetroxide in a postincubation step ${ }^{15}$ ).

After each procedure, scaffolds were dehydrated in an ascending series of ethanol and critical point dried in a Polaron E3000 apparatus (Quorum Technologies, Newhaven, United Kingdom) using liquid $\mathrm{CO}_{2}$.

\section{$C T$-scan imaging and data analysis}

The samples were scanned using the SkyScan 1072 MicroCT (SkyScan, Kontich, Belgium) with Feldkamp cone-beam reconstruction. NRecon V1.4.0 software was provided by SkyScan. The sample was mounted on a rotation stage in front of the X-ray source with Optosil (Heraeus, Armonk, NY). A high-resolution charge-coupled device with a resolution of $1024 \times 1024$ pixels was applied to detect the X-rays from the scanned samples and to store data. The following micro-CT settings were applied based on a pilot experiment: (1) the X-ray source was set to $47 \mathrm{kV}$ and $142 \mu \mathrm{A}$ resulting in a $6 \mu \mathrm{m}$ spot size, (2) a relatively high magnification $(125 \times$, pixel size: $2.26 \mu \mathrm{m}$ ) was applied to detect the structures of interest, (3) a relatively low exposure time (1.9s) was taken, (4) samples were rotated $180^{\circ}$ with a rotation step of $0.45^{\circ}$, and (5) a filter was omitted. An optical $1 \mathrm{~mm}^{3}$ selection was taken in the middle of the scaffold. After acquisition of the images at all rotation steps, the raw data were reconstructed to provide axial picture cross sections. After cone-beam reconstruction, the raw data were converted to a 16-bit bitmapped picture files with a resolution of $512 \times 512$ pixels. Using SkyScan's CT-analyzer v1.6 analyzing program, the pore size in the porous scaffolds and the distances between the lamellae in the unidirectional collagen samples were determined. Ten pores and the distance between 10 lamellae were measured automatically from a two-dimensional (2D) image at the largest diameter of the pore in the $X$ and $Y$ directions. 3D-Doctor V4.0 (Able Software, Lexington, MA) was used to create a final 3D model of the collagen sample. Model editing (e.g., rotating, editing, and transparency) was applied to optimize visualization of the collagen structures. Standardized software settings for scanning, cone-beam reconstruction, and gray-level thresholding were used in the projected and reconstructed files.

\section{Scanning electron microscopy}

Collagen scaffolds were mounted on stubs and sputtered with an ultrathin layer of gold in a Polaron E5100 Coating System (Quorum Technologies). Collagen scaffolds were studied with a Jeol (Tokyo, Japan) JSM-6310 SEM at an accelerating voltage of $15 \mathrm{kV}$.

\section{Light microscopy}

Scaffolds were fixed in $2.5 \%$ glutaraldehyde and $1 \%$ osmium tetroxide in $0.1 \mathrm{M}$ phosphate buffer ( $\mathrm{pH} 7.4)$ and embedded in epon. Sections of $\sim 1 \mu \mathrm{m}$ thickness were cut with an ultramicrotome, mounted on glass slides, and stained with $2 \%(\mathrm{w} / \mathrm{v})$ toluidine blue in $5 \%(\mathrm{w} / \mathrm{v})$ aqueous sodium tetraborate $10 \mathrm{H}_{2} \mathrm{O}$ for $20 \mathrm{~s}$. Sections were examined and photographed with a Leica (Wetzlar, Germany) DM 6000B light microscope.

\section{Results}

Two structurally different types of scaffolds were analyzed: scaffolds with large $(\sim 100 \mu \mathrm{m})$ round pores and scaffolds with unidirectional lamellae. The samples were rotated $180^{\circ}$ with a scanning period of $0.45^{\circ} / 1.9 \mathrm{~s}$ without the use of an additional filter. The average scanning time per sample was $1 \mathrm{~h}$. 
Table 1. Summary of Different Contrast Procedures and Their Effect on Micro-CT Contrast EnHancement of Collagenous Scaffolds

\begin{tabular}{|c|c|c|c|}
\hline Contrast agent(s) & Percentage applied & Impregnation time & Contrast \\
\hline Osmium tetroxide & $1 \%$ & 1 day & Weak \\
\hline Osmium tetroxide & $1 \%$ & 6 days & Moderate \\
\hline Lead citrate & $1 \%$ & 1 day & Weak \\
\hline Uranyl acetate & $2 \%$ & 1 day & Moderate \\
\hline Uranyl acetate + lead citrate & $2 \%+1 \%$ & 1 day +1 day & Good \\
\hline Osmium tetroxide + lead citrate & $1 \%+1 \%$ & 6 days +1 day & Moderate \\
\hline Osmium tetroxide + uranyl acetate & $1 \%+2 \%$ & 6 days +1 day & Good \\
\hline Osmium tetroxide + thiocarbohydrazide + osmium tetroxide & $1 \%+1 \%+1 \%$ & 6 days $+1 \mathrm{~h}+1 \mathrm{~h}$ & Moderate \\
\hline
\end{tabular}

Scaffolds with random round pores were visualized with different contrast enhancement techniques. Table 1 gives an overview of the protocols used and the contrast obtained as determined by visual inspection. Without any contrasting method, collagen scaffolds could not be visualized (data not shown). Moderate contrast enhancement in combination with a homogeneous distribution was achieved by the use of $2 \%$ uranyl acetate for 1 day, whereas the use of $1 \%$ osmium tetroxide for 1 day resulted in a lower signal, thus lower contrast (Fig. 1B, E). However, when scaffolds were im- pregnated with osmium tetroxide for 6 days, moderate contrast was achieved. In addition, thiocarbohydrazide was used to produce more contrast, but still only moderate contrast was obtained. The use of lead citrate gave poor results. A combination of contrast agents proved to be effective. The most suitable contrast techniques for visualization of collagen scaffolds were a combination of $1 \%$ osmium tetroxide for 6 days and $2 \%$ uranyl acetate for 1 day (Fig. 1A, D) or a combination of $2 \%$ uranyl acetate for 1 day and $1 \%$ lead citrate for 1 day. These good contrast methods lead to most
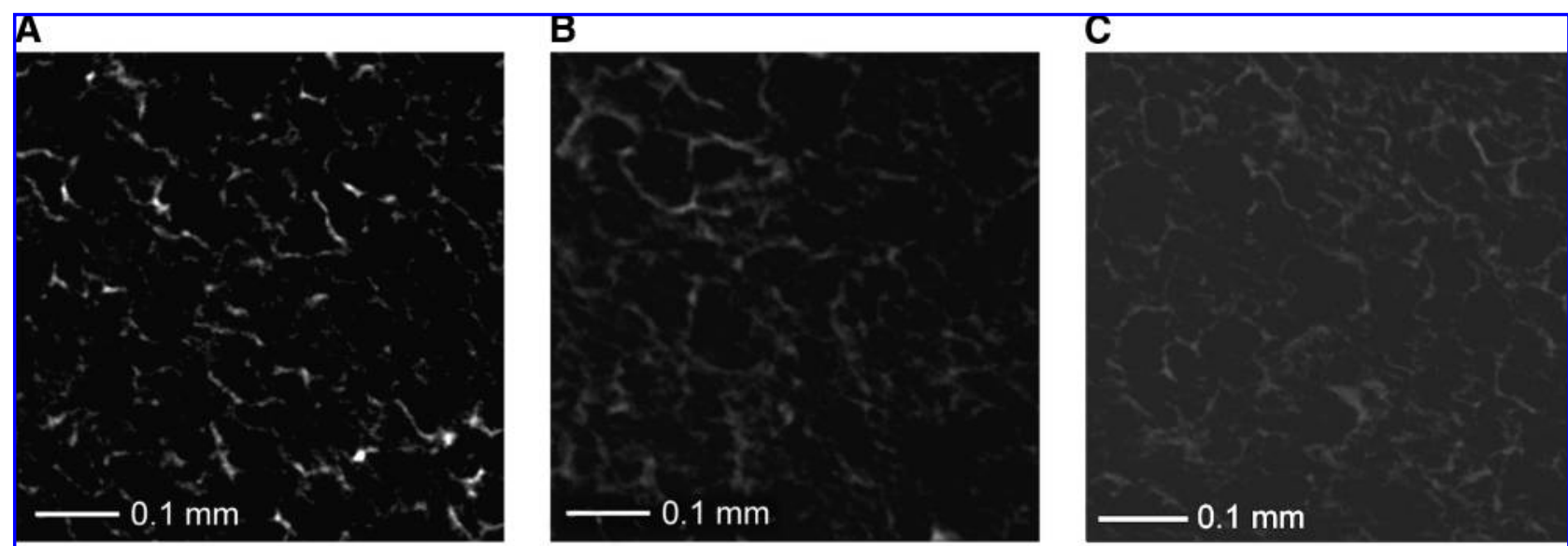

D

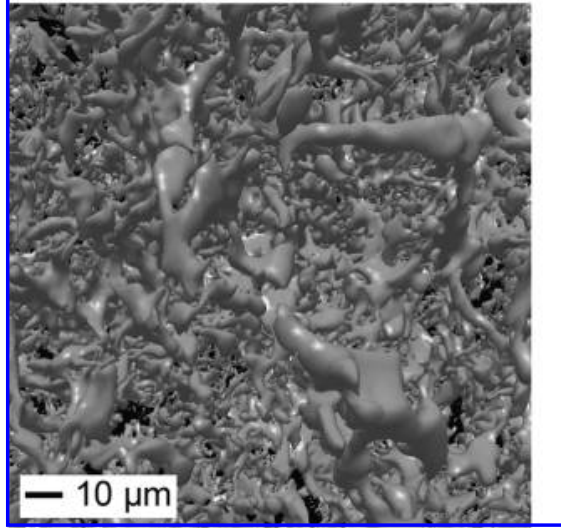

E

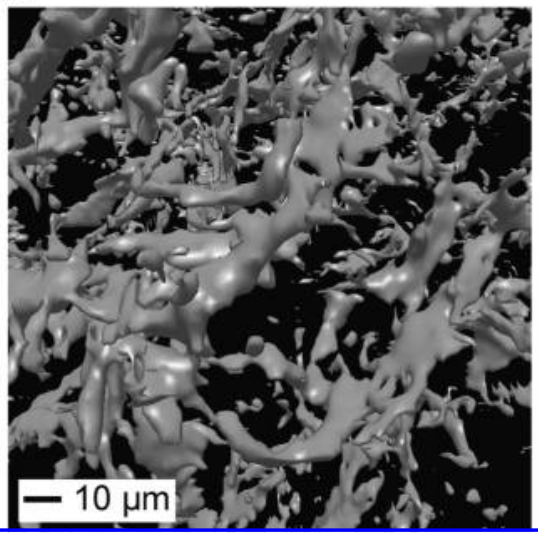

$\mathbf{F}$

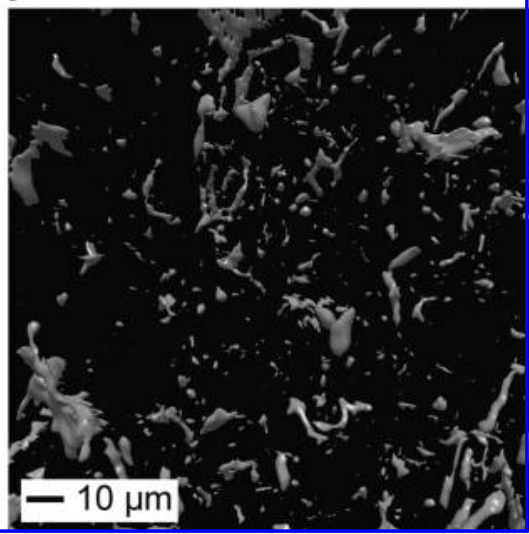

FIG. 1. Effect of three contrast procedures on the attenuation of X-rays by porous collagenous scaffolds in a micro-CT setting. (A-C) represent 2D images, whereas (D-F) represent 3D images. (A, D) 1\% osmium tetroxide for 6 days followed by $2 \%$ uranyl acetate for 1 day; $(\mathbf{B}, \mathbf{E}) 1 \%$ osmium tetroxide for 6 days; $(\mathbf{C}, \mathbf{F}) 1 \%$ osmium tetroxide for 1 day. Note good contrast in $(\mathbf{A}, \mathbf{D})$, moderate contrast in $(\mathbf{B}, \mathbf{E})$, and poor contrast in $(\mathbf{C}, \mathbf{F})$. 


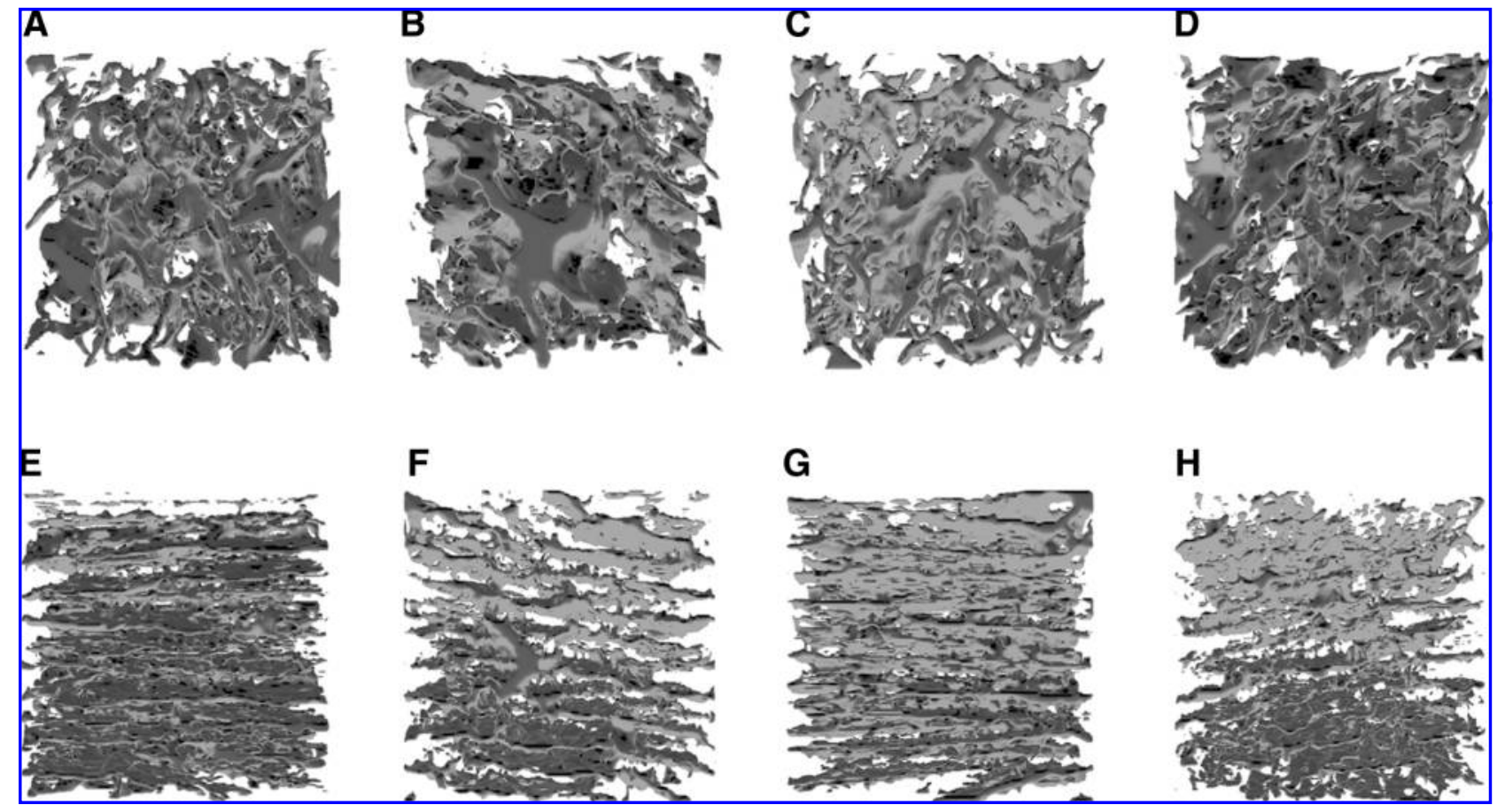

FIG. 2. Snapshot still images taken from (A-D) a porous scaffold rotated around its $y$-axis and (E-H) a unidirectional scaffold rotated around its $x$-axis.
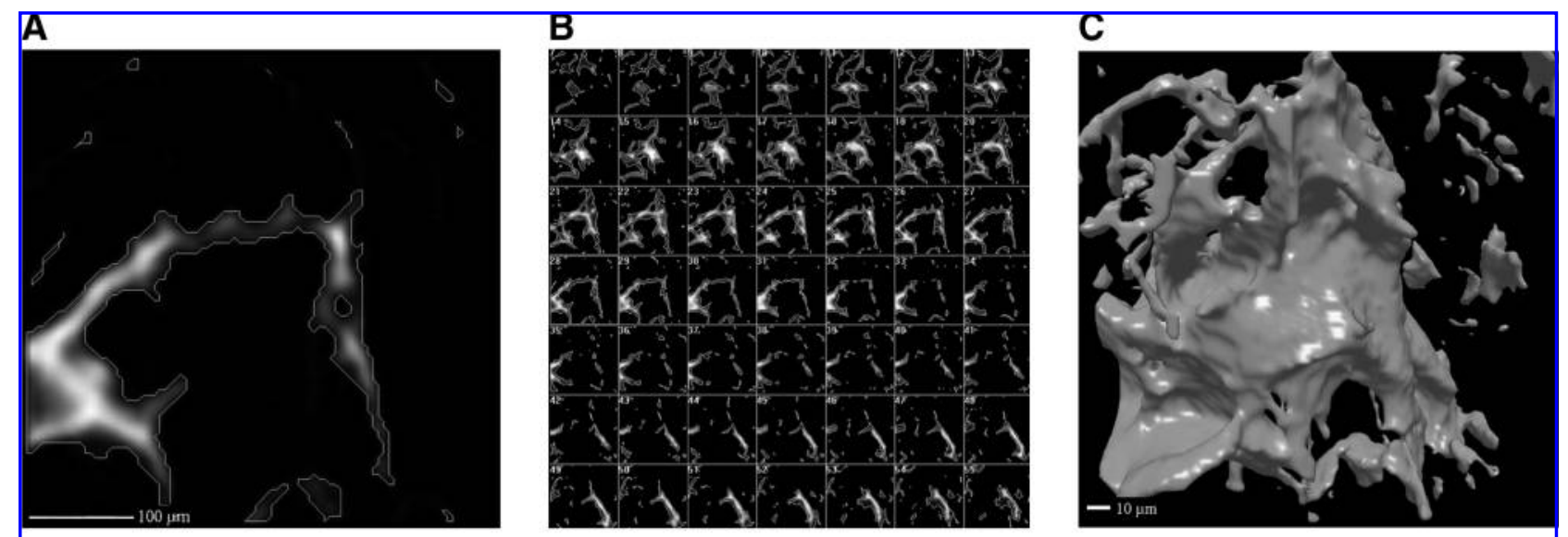

D

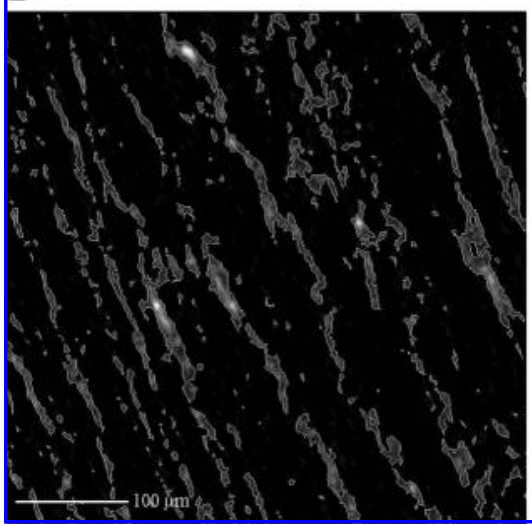

\section{$\mathbf{E}$}

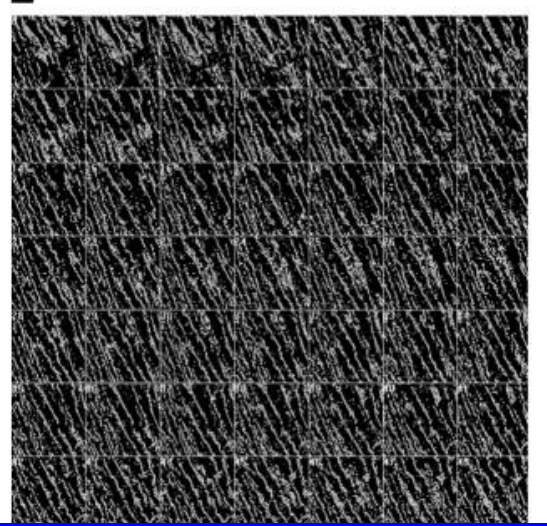

\section{$\mathbf{F}$}

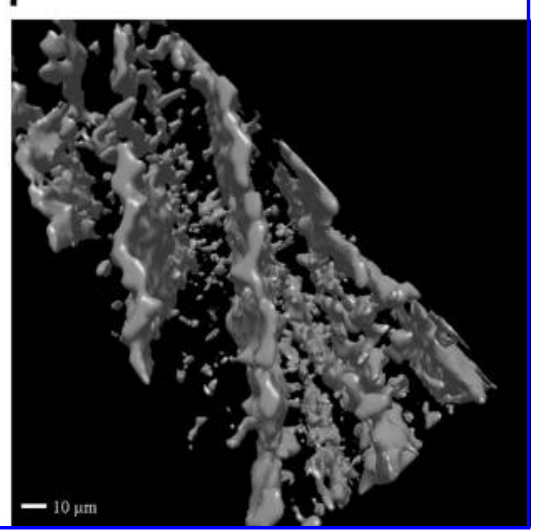

FIG. 3. Micro-CT analysis of two different contrast-enhanced collagenous scaffolds. (A-C) Scaffold with round pores; (D, E) scaffold with unidirectional lamellae (D-F). (A, D) Single 2D projection image of a representative part of the scaffold; (B, E) complete stack of 2D images, covering $225 \mu \mathrm{m} ;(\mathbf{C}, \mathbf{F})$ final 3D reconstruction showing porous and lamellar structures, respectively. Round pore diameters are $\sim 100 \mu \mathrm{m}$, whereas distances between the lamellae are $\sim 50 \mu \mathrm{m}$. Contrast method: $1 \%$ osmium tetroxide for 6 days and $2 \%$ uranyl acetate for 1 day. Scale bars are $100 \mu \mathrm{m}$ in (A, D) and $10 \mu \mathrm{m}$ in $(\mathbf{C}, \mathbf{F})$. 
detail in the $3 \mathrm{D}$ reconstructions, whereas a weak contrasting protocol resulted in a poor $3 \mathrm{D}$ reconstruction (Fig. 1C, F).

Two movies (see Supplemental Movies roundpores.avi and unidirectionallamellae.avi, available online at www .liebertonline.com) show the 3D reconstruction of the collagen scaffolds with round pores and of the unidirectional lamellae from different angles rotated around the $x$-axis and $y$-axis. Figure 2 is a compilation of snapshot images from these movies. Figure 2A-D indicates random porosity of the porous scaffold, whereas Figure 2E-H points out the unidirectionality of the unidirectional scaffold.

Using contrasting protocol 6, initial measurements were made, and the pore diameter could be determined. A representative part of the whole samples of collagen scaffolds with round pores and unidirectional lamellae was reconstructed using a stack of 2D images (see Fig. 3B for a scaffold with round pores and Fig. 3E for a unidirectional scaffold). Micro$\mathrm{CT}$ images of collagen scaffolds with round pores revealed pore sizes of $\sim 100 \mu \mathrm{m}$ (Fig. 3A), whereas the distance between the lamellae in unidirectional scaffolds was around $50 \mu \mathrm{m}$ (Fig. 3D). With the use of the whole file series of all gray values from the scaffold material compared to the radio opaque air, the porosity of the scaffold was estimated to be $86 \pm 5 \%(n=5 \pm \mathrm{SD})$. Finally, the data obtained by micro-CT were compared to data acquired by scanning electron microscopy and by light microscopy. The pore diameter in scaffolds with round pores was generally about $100 \mu \mathrm{m}$ for both methodologies, similar to micro-CT data (Fig. 4A-C). Scaffolds with unidirectional lamellae examined with scanning electron microscopy showed a distance between the la- mellae of $51 \pm 11 \mu \mathrm{m}(n=50 \pm \mathrm{SD})$, similar to micro-CT. Using light microscopy, this distance was $64 \pm 9 \mu \mathrm{m}(n=50 \pm \mathrm{SD})$ (Fig. 4D-F). However, the micro-CT image of the unidirectional scaffold did not reflect the complete structure of the scaffold; the small struts in between the lamellae (generally $1-2 \mu \mathrm{m})$ were too thin to be visualized, because they are below the resolution limit of the equipment, which is about $6 \mu \mathrm{m}$ using the applied settings.

\section{Discussion}

The 3D architecture of the scaffold is of great importance for the behavior of cells. Cells behave differently in a 3D environment compared to a $2 \mathrm{D}$ structure. ${ }^{16-19}$ Therefore, effective scaffold assessment techniques are required to evaluate the structural characteristics of scaffolds. Among these techniques SEM analysis is most popular for soft tissue, whereas micro-CT is generally used for hard tissue (such as bone). ${ }^{3-5,10}$

We here show imaging of collagen scaffolds by means of micro-CT. Using metal-based contrast enhancement methods, the structure of collagen scaffolds was visualized. In this study, we only looked at porosity, but with the appropriate software, it is possible to determine other parameters, like density and interconnectivity, for soft scaffolds. When the structures in the collagen scaffolds are not too small, the 3D data sets obtained using micro-CT provide information about the sample's structure that is comparable to complementary methods such as light and electron microscopy, but with the obvious advantage of $3 \mathrm{D}$ of the whole specimen. ${ }^{20-23}$ We were
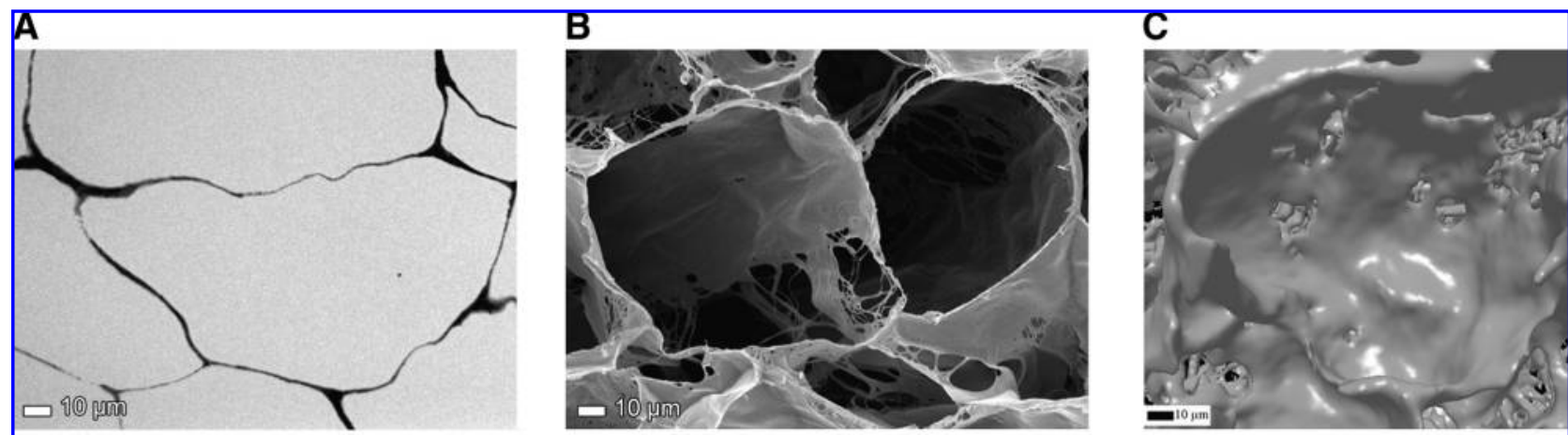

D

E

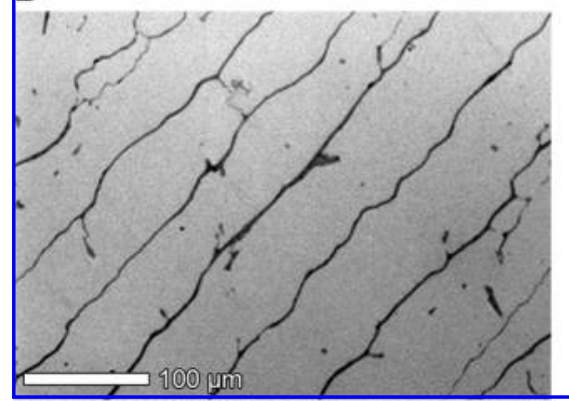

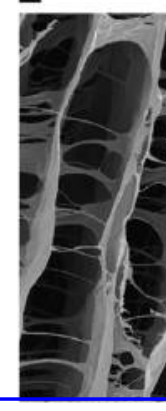

$\mathbf{F}$

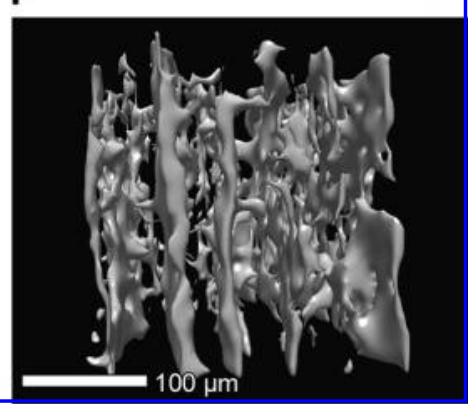

FIG. 4. Comparison of images obtained using micro-CT with those obtained by light and electron microscopy pictures of random (A-C) and unidirectional (D-F) collagen scaffold obtained with light microscopy (A, D), scanning electron microscopy (B, E), and micro-CT imaging $(\mathbf{C}, \mathbf{F})$. Note comparable morphology between different analysis techniques. Also note that the fine struts in between collagen lamellae are not visible in the micro-CT image. Scale bars are $10 \mu \mathrm{m}$ in $(\mathbf{A}-\mathrm{C})$ and $100 \mu \mathrm{m}$ in $(\mathbf{D}-\mathbf{F})$. 
unable to visualize collagen scaffolds without contrast agents. Buttafoco et al. ${ }^{24}$ did succeed in micro-CT visualization of collagen-elastin tubes without contrast agents. This difference with our scaffolds cannot be explained because the presence of thick elastin fibers does not provide more image contrast.

When structures are too small, one encounters the instrumental limitations of the micro-CT equipment. The estimated porosity may thus be overvalued because of this. Nano-CT may be a solution to this problem. The theoretical instrumental limitation of the nano-CT apparatus is $200 \mathrm{~nm}$ compared to $2 \mu \mathrm{m}$ for the micro-CT apparatus. ${ }^{25}$

Drawbacks of micro-CT are the difficulty of thresholding, beam hardening, and the need for specialized software to quantify certain parameters (e.g., interconnectivity). ${ }^{6}$ Before $3 \mathrm{D}$ modeling, the crucial step is to separate scaffold material from background (performed by thresholding the image gray level ${ }^{9}$ ), which affects the subsequent visualization. When too much thresholding is applied, scaffold material is undervalued. Beam hardening is known as the high exposure of the scaffold center as a result of scaffold attenuation of the lower energy rays of the used polychromatic X-ray beam. As a consequence, thresholding is no longer dependent solely on radiodensity, but also on specimen size. ${ }^{26,27}$

Although micro-CT still faces some problems when used for scaffolds made from soft natural biomaterials (i.e., limitation to visualize small structures), this method is able to provide a $3 \mathrm{D}$ reconstruction of the specimen in little time and with little processing, whereas techniques like scanning electron microscopy and light microscopy are very laborious. In addition, it is possible to scan a large specimen with good morphology of the whole scaffold. It should be seen as complementary to other methods.

\section{Conclusion}

Using specific contrasting protocols, we showed the potential of imaging soft, proteinaceous materials by micro-CT. The most suitable contrast techniques for visualization of collagen scaffolds were a combination of $1 \%$ osmium tetroxide for 6 days $+2 \%$ uranyl acetate for 1 day, and a combination of $2 \%$ uranyl acetate for 1 day $+1 \%$ lead citrate for 1 day. A drawback is that small details (less than few $\mu \mathrm{m}$, e.g., struts in unidirectional scaffolds) cannot be observed with micro-CT. For better resolution, nano-CT is needed.

\section{Acknowledgments}

This work was financially supported by EU-FP6 project EuroSTEC (soft tissue engineering for congenital birth defects in children; contract: LSHB-CT-2006-037409) and the Dutch Program for Tissue Engineering (DPTE6735).

\section{Disclosure Statement}

No competing financial interests exist.

\section{References}

1. Guldberg, R.B. Tissue engineering: micro-CT imaging. Sci Med 9, 9, 2003.

2. Feldkamp, L.A., Goldstein, S.A., Parfitt, A.M., Jesion, G., and Kleerekoper, M. The direct examination of three- dimensional bone architecture in vitro by computed tomography. J Bone Miner Res 4, 311, 1989.

3. Muller, R., Hildebrand, T., and Ruegsegger, P. Non-invasive bone biopsy: a new method to analyse and display the threedimensional structure of trabecular bone. Phys Med Biol 39, 145, 1994.

4. Ruegsegger, P., Koller, B., and Muller, R. A microtomographic system for the nondestructive evaluation of bone architecture. Calcif Tissue Int 58, 24, 1996.

5. Goulet, R.W., Goldstein, S.A., Ciarelli, J.L, Brown, M.B., and Feldkamp, L.A. The relationship between the structural and orthogonal compressive properties of trabecular bone. J Biomech 27, 375, 1994.

6. Ho, S.T., and Hutmacher, D.W. A comparison of micro-CT with other techniques used in the characterization of scaffolds. Biomaterials 27, 1362, 2006.

7. Lin, A.S.P., Barrows, T.H., Cartmell, S.H., and Guldberg, R.E. Microarchitectural and mechanical characterization of oriented porous polymer scaffolds. Biomaterials 24, 481, 2003.

8. Lin-Gibson, S., Copper, J.A., Landis, F.A., and Cicerone, M.T. Systematic investigation of porogen size and content on scaffold morphometric parameters and properties. Biomacromolecules 8, 1511, 2007.

9. Peyrin, F., Mastrogiacomo, M., and Cancedda, R. SEM and 3-D synchrotron radiation. Micro-tomography in the study of bioceramic scaffolds for tissue-engineering applications. Biotechnol Bioeng 9, 638, 2007.

10. Wang, F., Shor, L., Darling, A., Khalil, S., Sun, W., Guceri, S., and Lau, A. Precision extruding deposition and characterization of cellular poly- $\varepsilon$-lactone tissue scaffolds. Rapid Prototyping J 10, 42, 2004.

11. Faraj, K.A., van Kuppevelt, T.H., and Daamen, W.F. Construction of collagen scaffolds that mimic the threedimensional architecture of specific tissues. Tissue Eng 13, 2387, 2007.

12. Schoof, H., Apel, J., Heschel, I., and Rau, G. Control of pore structure and size in freeze-dried collagen sponges. J Biomed Mater Res 58, 352, 2001.

13. Ann Ellis, A. Poststaining grids for transmission electron microscopy conventional and alternative protocols. Methods Mol Biol 369, 97, 2007.

14. Kelly, R.O., Dekker, R.A., and Bleumink, K. Ligandmediated osmium binding: its application in coating biological specimens for scanning electron microscopy. J Utrastruct Res 45, 254, 1973.

15. Seligman, A.M., Wasserkrug, H.L., and Hanker, J.S. A new staining method (OTO) for enhancing contrast in lipidcontaining membranes and droplets in osmium tetraoxidefixed tissue with osmiophilic thiocarbohydrazide (TCH). J Cell Biol 30, 424, 1996.

16. Inanc, B., Elcin, A.E., and Elcin, Y.M. Osteogenic induction of human periodontal ligament fibroblasts under two- and three-dimensional culture conditions. Tissue Eng 12, 257, 2006.

17. Sun, T., Jackson, S., Haycock, J.W., and MacNeil, S. Culture of skin in 3-D rather than 2D improves their ability to survive exposure to cytotoxic agents. J Biotechnol 122, 372, 2006.

18. Stegemann, J.P., and Nerem, R.M. Altered response of vascular smooth muscle cells to exogenous biochemical stimulation in two- and three-dimensional culture. Exp Cell Res 283, 146, 2003.

19. Cukierman, E., Pankov, R., Stevens, D.R., and Yamada, K.M. Taking cell-matrix adhesions to the third dimension. Science 294, 1708, 2001. 
20. Ritman, E.L. Micro-computed tomography-current status and developments. Annu Rev Biomed Eng 6, 185, 2004.

21. van Oosterwyck, H., van Der Sloten, J., van Der Perre, G., Jansen, J., Wevers, M., and Naert, I. Use of microfocus computerized tomography as a new technique for characterizing bone tissue around oral implants. J Oral Implantol 26, 5, 2000.

22. Gauthier, O., Muller, R., Von Stechow, D., Lamy, B., Weiss, P., Bouler, J.M., Aguado, E., and Daculsi, G. In vivo bone regeneration with injectable calcium phosphate biomaterial: a three-dimensional micro-computed tomographic, biomechanical and SEM study. Biomaterials 26, 5444, 2005.

23. Cartmell, S., Huynh, K., Nagaraja, S., and Guldberg, R. Quantitative microcomputed tomography analysis of mineralization within three-dimensional scaffolds in vitro. J Biomed Mater Res A 69, 97, 2004.

24. Buttafoco, L., Engbers-Buijtenhuijs, P., Poot., A.A., Dijkstra, P.J., Vermes, I., and Feijen, J. Physical characterization of vascular grafts cultured in a bioreactor. Biomaterials 11, 2380, 2006.

25. Cnudde, V., Masschaele, B., Dierick, M., Vlassenbroeck, J., van Hoorebeke, L., and Jacobs, P. Recent progress in X-ray CT as a geosciences tool. Appl Geochem 21, 826, 2006.
26. Duvall, C.L., Taylor, W.R., Weiss, D., and Guldberg, R.E. Quantitative microcomputed tomography analysis of collateral vessel development after ischemic injury. Am J Physiol Heart Circ Physiol 287, H302, 2004.

27. Bonse, U., and Busch, F. X-ray computed microtomography (microCT) using synchrotron (SR). Prog Biophys Mol Biol 65, 133, 1996.

Address correspondence to: Willeke F. Daamen, Ph.D.

Department of Biochemistry 280 Nijmegen Centre for Molecular Life Sciences Radboud University Nijmegen Medical Centre

P.O. Box 9101

6500 HB Nijmegen

The Netherlands

E-mail: w.daamen@ncmls.ru.nl

Received: July 29, 2008

Accepted: January 20, 2009

Online Publication Date: March 3, 2009 



\section{This article has been cited by:}

1. Chelsea L. Gregg, Jonathan T. Butcher. 2012. Quantitative in vivo imaging of embryonic development: Opportunities and challenges. Differentiation 84:1, 149-162. [CrossRef]

2. Sean M. White, Ryan Hingorani, Rajan P.S. Arora, Christopher C.W. Hughes, Steven C. George , Bernard Choi . Longitudinal In Vivo Imaging to Assess Blood Flow and Oxygenation in Implantable Engineered Tissues. Tissue Engineering Part C: Methods, ahead of print. [Abstract] [Full Text HTML] [Full Text PDF] [Full Text PDF with Links] [Supplemental material]

3. Dr. Sean White, Mr. Ryan Hingorani , Mr. Rajan Arora , Prof. Christopher C.W. Hughes , Prof. Steven George , Dr. Bernard Choi . Longitudinal in vivo imaging to assess blood flow and oxygenation in implantable engineered tissues. Tissue Engineering Part C: Methods 0:ja. . [Abstract] [Full Text PDF] [Full Text PDF with Links]

4. Willeke Daamen, Kaeuis Faraj, Martin Koens, Gerwen Lammers, Katrien Brouwer, Peter Uijtdewilligen, Suzan Nillesen, Luc Roelofs, Jody Nuininga, Paul Geutjes, Wouter Feitz, Toin van KuppeveltExtracellular Matrix-Based Scaffolds from Scratch 385-398. [CrossRef]

5. Vinay M. Pai, Megan Kozlowski, Danielle Donahue, Elishiah Miller, Xianghui Xiao, Marcus Y. Chen, Zu-Xi Yu, Patricia Connelly, Kenneth Jeffries, Han Wen. 2012. Coronary artery wall imaging in mice using osmium tetroxide and microcomputed tomography (micro-CT). Journal of Anatomy no-no. [CrossRef]

6. Alyssa Appel, Mark A. Anastasio, Eric M. Brey . 2011. Potential for Imaging Engineered Tissues with X-Ray Phase Contrast. Tissue Engineering Part B: Reviews 17:5, 321-330. [Abstract] [Full Text HTML] [Full Text PDF] [Full Text PDF with Links]

7. Ryuta Mizutani, Yoshio Suzuki. 2011. X-ray microtomography in biology. Micron . [CrossRef]

8. Nathan S. Jeffery, Robert S. Stephenson, James A. Gallagher, Jonathan C. Jarvis, Philip G. Cox. 2011. Micro-computed tomography with iodine staining resolves the arrangement of muscle fibres. Journal of Biomechanics 44:1, 189-192. [CrossRef]

9. Eric M. Brey, Alyssa Appel, Yu-Chieh Chiu, Zhong Zhong, Ming-Huei Cheng, Holger Engel, Mark A. Anastasio . 2010. X-Ray Imaging of Poly(Ethylene Glycol) Hydrogels Without Contrast Agents. Tissue Engineering Part C: Methods 16:6, 1597-1600. [Abstract] [Full Text HTML] [Full Text PDF] [Full Text PDF with Links] 